\title{
A multidisciplinary team approach to airway management in a patient with subglottic stenosis: advantages and limitations of videolaryngoscopy and considerations for effective surgical airway management
}

Jason D. Walls, MD'; Ankona Ghosh, MD²; and Joshua H. Atkins, MD, PhD ${ }^{1}$

Emergent airway management in the Intensive Care Unit (ICU) presents a unique challenge and difficult intubation is more common in this setting compared to the controlled conditions found in the operating room. Clinicians have come to rely on videolaryngoscopy to facilitate airway management while perhaps underestimating the potential for failure. We present a case of a patient requiring tube exchange in the ICU after witnessed cardiac arrest. Common approaches to tube exchange, including videolaryngoscopy failed due to subglottic stenosis. Prompt recognition and activation of the hospital-wide surgical airway team facilitated discussion and successful tracheostomy placement at the bedside.

Translational aspect of this case report: A multidisciplinary team approach with shared medical decision-making is critical during acute airway management especially in out of OR settings.

\section{Introduction}

Difficult airway situations are rare events occurring in only $1.8-5.8 \%$ of all intubations, but can be as frequent as $6.6-22 \%$ in critically ill patients. ${ }^{1}$ The American Society of Anesthesiologists recently updated Practice Guidelines for the Management of the Difficult Airway and for the first time recommended consideration of video-assisted laryngoscopy as a first line approach for potentially challenging intubations. ${ }^{2}$ However, videolaryngoscopy is met with failure in a finite percentage of cases, including tracheal stenosis. Although not able to facilitate tracheal intubation in these situations, videolaryngoscopy can still be helpful in airway assessment. Ultimately, the surgical airway is the definitive intervention in the face of failed intubation in the emergency setting. During these times of crisis, reliable dissemination of information and efficient resource allocation is crucial. Implementation of systems-based solutions such as the airway rapid response team and difficult airway bracelet system can facilitate care in emergent settings.

\section{Case Description}

The on-call anesthesia team was urgently summoned to the medical ICU of a large academic medical center to evaluate a patient with an "unstable" endotracheal tube that was concerning for imminent self-extubation. On arrival to the bedside, a covering physician with limited familiarity of the patient gave report. Since the patient had just been admitted, the paper records from the emergency department (ED) with information regarding his airway management were unavailable. The patient was a 60 yearold man of average height and weight who presented to the ED earlier that day in cardiac arrest secondary to hypoxia caused by a severe exacerbation of underlying chronic obstructive pulmonary disease. In the ED, he was resuscitated, intubated by the emergency room physicians with difficulty using a $6.0 \mathrm{~mm}$ endotra- cheal tube (ETT), and received bilateral thoracostomy tubes for presumed bilateral tension pneumothoraxes. Due to the lack of a neurological exam after intubation, he was cooled according to the hospital hypothermia protocol for witnessed cardiac arrest.

On initial inspection, the patient had no overt predictors of a difficult airway. His mentohyoid and thyromental distances were greater than $3 \mathrm{~cm}$, neck circumference was $<35 \mathrm{~cm}$, mouth opening was not constrained, and there were no other anatomic features indicating difficult mask ventilation or intubation. The patient was sedated on a fentanyl infusion and nonreactive to external stimulation. He was hemodynamically stable with an oxygen saturation of $96-100 \%$ on $100 \%$ inspired oxygen. A 6.0 $\mathrm{mm}$ ETT tube was in place and taped at $19 \mathrm{~cm}$ at the lip. Exhaled tidal volumes on assist control ventilation varied from 100-200 $\mathrm{mL}$ and bilateral air-leaks were present. Arterial blood gas values ( $\mathrm{pH} 7.34, \mathrm{PCO}_{2} 34 \mathrm{mmHg}, \mathrm{PaO} 2378 \mathrm{mmHg}, 100 \% \mathrm{FIO}_{2}$ ) were consistent with adequate ventilation.

Direct laryngoscopy with a Glidescope ${ }^{\circledR}$ (Verathon, Inc. Bothell, WA) MAC 3 blade produced a Grade I view of the glottis and revealed that the cuff of the 6.0 ETT had herniated above the vocal cords. The cuff was deflated and an attempt to advance the tube resulted in coiling in the posterior oropharynx. Under direct vision, the tube was removed over a soft-tipped extra-firm 14 Fr/100cm Cook exchange catheter (Cook Medical, Bloomington, IN), but over-catheter exchange attempts with a $8.0 \mathrm{~mm}$ and $7.0 \mathrm{~mm}$ ETT both encountered resistance just below the glottis opening. A fresh $6.0 \mathrm{~mm}$ ETT could be advanced no further than the glottic inlet where stiff resistance was met. The cuff was re-inflated, the exchange catheter removed and positive pressure ventilation re-initiated. At this time, an Airway Rapid Response was activated which promptly brings a trauma surgery attending, senior otolaryngology (ENT) house staff, and surgical airway equipment, including a tracheostomy tray to the bedside. With this team assembled at the bedside, the 6.0 ETT was removed over a tube exchange catheter and the ENT surgeons performed fiberoptic bronchoscopy with a pediatric scope. This revealed a $>50 \%$ subglottic stenosis caused by an anterior shelf of granulation spanning at least $2 \mathrm{~cm}$ below the vocal cords and extending distally. The decision was made to attempt passage of a 5.0 microlaryngeal tracheal tube (MLT ${ }^{\circledR}$, Covidien, Waltham, MA) over the rigid tube exchanger as a final non-invasive attempt. This also met resistance. The $5.0 \mathrm{~mm}$ ETT was advanced as far as possible, the cuff-inflated and positive pressure ventilation reinitiated. Due to the balloon inflation at the level of the cords, a seal could be obtained for positive pressure ventilation, but intermittently dislodged with movement of the patient and was inadequate for long-term ventilation. The team agreed that a surgical airway was indicated.

A multidisciplinary discussion between the anesthesia, ENT, and trauma surgery attendings, as well as nursing staff ensued regarding the type of surgical airway to perform and the optimal location for the procedure. Consideration was given to transport 
to the operating room for the procedure. However, the complexity of the patient's condition, including the tenuous nature of the airway, the need for continuous negative pressure on the bilateral thoracostomy tubes, the inability to reliably measure exhaled tidal volumes due to the bronchopleural fistula (BPF), and the equipment in place to maintain therapeutic hypothermia, resulted in a team decision to proceed with an open bedside tracheostomy in the ICU. To optimize the conditions, a surgical nursing team and additional surgical equipment, including electrocautery, were brought to the ICU. A tracheostomy was performed at the bedside using a standard midline approach at a level approximately half the distance between the cricoid cartilage and sternal notch. The patient was provided anesthesia via fentanyl infusion. A $6.0 \mathrm{~mm}$ cuffed Shiley ${ }^{\mathrm{TM}}$ tracheostomy tube (Covidien, Waltham, MA) could not be passed through the distal stenotic areas but a $4.0 \mathrm{~mm}$ cuffed Shiley ${ }^{\mathrm{TM}}$ tracheostomy tube met no resistance. Position was confirmed by colorimetric $\mathrm{CO} 2$ analysis and fiberoptic tracheoscopy. The oxygen saturation was greater than $90 \%$ throughout the airway management process. The patient expired 48 hours after the procedure when life supportive measures were withdrawn by the family due to lack of neurological recovery after rewarming.

\section{Discussion}

This case highlights the confluence of a number of critical aspects of emergent airway management outside of the operating room. The evolution of the case, the complicated medical presentation, and the decision making process described highlight the need for open communication, teamwork, and efficient resource allocation. The case also reinforces the potential for systems failures, especially in communication, that complicate patient management.

Initially, this patient presented a complicated picture, especially in evaluating the integrity of his ETT. The decreased and varied tidal volumes heralded a problem thought to be related to bilateral BPF. Iatrogenic BPF is a known complication of tube thoracostomy. ${ }^{3}$ This is detected by persistent air-leak during positive pressure ventilation. The loss of ventilated gas complicates ventilator management including the inability to reliably measure delivered minute ventilation. The bilateral BPF in this patient severely reduced the measured exhaled tidal volumes, which in conjunction with the shallow position of an overtly mobile endotracheal tube presented a confusing picture to the clinicians caring for the patient.

A detailed post-event review of the emergency department records revealed difficulty with repeated attempts at intubation with a larger bore tube and tube exchange over a catheter. As stated above, this information was not available to the emergency responders. This health system currently has a difficult airway bracelet system to provide a global indicator to all providers about the potential need for special techniques in airway management. Additionally, a form is placed above the bed with a detailed description of successful and unsuccessful airway interventions. However, this bracelet placement was not performed in the ED and subsequent placement in the ICU would require an urgent consult to anesthesiology for evaluation. Since the patient was already intubated, the process had not yet been initiated for this patient. This is further testimony to the fact that even well designed critical alert systems can fail in unusual circumstances.

In addition, the initial approach was to visualize the glottis with the Glidescope ${ }^{\circledR}$ video laryngoscope. The Glidescope ${ }^{\circledR}$ has been proven to improve glottic visualization, especially in patients with suspected difficult airways. ${ }^{1}$ Despite the lack of literature demonstrating superiority of this technique to standard methods, there is a developing trend toward videolaryngoscopy as a first-line intubation approach for critically ill. ${ }^{4}$ The Glidescope ${ }^{\circledR}$ has an overall success rate of $96 \%$ with several preoperative predictors of failure including neck pathology, short thyromental distance, decreased cervical motion, and distal tracheal disease. Age, gender, BMI, oral opening, and Mallampati score are not associated with failed videolaryngoscopy. ${ }^{5}$ Videolaryngoscopy is associated with improved glottic view in many studies but as evidenced in this case, optimal glottic visualization does not always guarantee successful tracheal intubation. In this case, the videolaryngoscope provided a continuous view of the glottis to the entire team that was an extremely useful tool for collaborative management and decision-making. The team was able to immediately diagnosis the inadequate tube position, confirm that the limitation to tube passage was distal airway obstruction rather than laryngeal pathology or arytenoid interference, and also afforded the team direct visualization of attempts at catheter guided tube exchange. In the absence of continuous capnography and reliable exhaled tidal volumes, the Glidescope ${ }^{\circledR}$ also allowed direct confirmation of appropriate tube placement in the tracheal during interval changes with repeated intubation attempts.

This case also highlights the limitations of Glidescope ${ }^{\circledR}$ technology with regard to intubation with special tubes. Glidescope ${ }^{\circledR}$ intubation is most reliably accomplished with the GlideRite ${ }^{\circledR}$ stylet, the specially shaped stylet provided by the manufacturer. This stylet cannot pass through tube sizes smaller than $5.0 \mathrm{~mm}$ and does not reach the distal tip when using an MLT type tube. In such circumstances, intubation can be challenging, as it requires an experienced operator to manually fashion an appropriately molded stylet. Alternative technologies such as the Storz C-MAC laryngoscope do not utilize a preformed stylet and may provide for more space for maneuvering airway instruments during laryngoscopy, but few direct comparisons of efficacy among available devices have been reported. ${ }^{6}$

Although the Glidescope ${ }^{\circledR}$ has several limitations in situations of unexpected subglottic stenosis, the Glidescope ${ }^{\circledR}$ does have a utility is these situations for direct visualization of tube exchange. In a few case series, video laryngoscopy was a helpful adjunct to endotracheal tube exchange, especially in the high-risk patient with a known difficult airway. ${ }^{7}$ The video laryngoscope changes a blind procedure into one that allows full visualization of the glottic opening and direct visualization of tube exchange. This allows for rapid diagnosis of tube exchange difficulties and visualization of any pathology near the glottic opening. As illustrated by this case, the use of advanced video laryngoscope technology can provide adequate glottic visualization allowing for better understanding of the difficult airway etiology and rapid transition to the difficult airway algorithm and surgical airway.

Surgical airways outside of the operating room represent a significant practical challenge to assemble critical resources in a timely fashion. The difficult airway bracelet system is a systematic way to communicate known airway issues and pre-emptively trigger special approaches in the known difficult airway. ${ }^{8}$ Over three years ago, this institution implemented an airway rapid response team that brings a designated set of resources, including airway surgeons and operating equipment to the bedside in a coordinated fashion. Systems designed to optimize local resources for airway emergencies have been reported. ${ }^{5}$ The case reported here highlights some subtle aspects of the surgical airway response that are worthy of consideration, specifically the decision to proceed with bedside tracheostomy. Initially, the trau-

Trans Periop \& Pain Med 2014 Volume 1 Issue 1 Page 15 
ma surgeon planned a cricothyrotomy as the approach to secure the airway consistent with recommendations of the American Trauma Life Support manual. ${ }^{9}$ However, the ENT surgeons noted that a more distal approach in a formal tracheostomy was indicated because a cricothyrotomy incision would be too proximal in the airway to allow for successful tube placement distal to the area of stenosis. Moreover, from the bronchoscopic view, it was evident that the patient would need the inevitable conversion to tracheotomy afterwards for long-term airway management. All team members acknowledged that a tracheostomy was a more technically challenging procedure, especially in the out-of-operating room setting, but was the best solution for long-term airway management.

\section{Conclusion}

Undiagnosed subglottic stenosis provides many challenges to intubation outside of the operating room. Use of video laryngoscope technology can aid in diagnosis and transition to a surgical airway. A multidisciplinary team approach with shared medical decision-making is critical during acute airway management.

\section{Conflict of Interest}

The authors declare no conflict of interests.

\section{Acknowledgements}

The authors acknowledge the helpful contributions of Linda Chen, MD during manuscript preparation.

\section{Address for correspondence}

Corresponding author: Joshua H. Atkins, Assistant Professor, Mailing address: Department of Anesthesiology and Critical Care, Perelman School of Medicine at the University of Pennsylvania, 6 Dullus, 3400 Spruce Street, Philadelphia PA, 19104 USA.

Email: Joshua.atkins@uphs.upenn.edu

\section{Disclosure of Funding}

This work is supported by departmental funding.

\author{
Additional publication details \\ Journal short name: Trans Periop \& Pain Med \\ Received Date:May 1, 2014 \\ Accepted Date: June 1, 2014 \\ Published Date: July 1, 2014 \\ Trans Periop \& Pain Med 2014, 1:13-15
}

\section{Citation and Copyrights}

Citation: Walls, Jason: A multidisciplinary team approach to airway management in a patient with subglottic stenosis: advantages and limitations of videolaryngoscopy and considerations for effective surgical airway management. Trans Periop \& Pain Med 2014, 1(1):14-16

Copyright: (c) 2014 Walls, D. Jason, et al. This is an open-access article distributed under the terms of the Creative Commons Attribution License, which permits unrestricted use, distribution, and reproduction in any medium, provided the original author and source are credited.

References

1. Griesdale DE, Liu D, McKinney J, Choi PT. Glidescope ${ }^{\circledR}$ video-laryngoscopy versus direct laryngoscopy for endotracheal intubation: a systematic review and meta-analysis. Can J Anaesth 2012; 59(1):41-52. $2 . \quad$ American Society of Anesthesiologists Task Force on Management of the Difficult Airway. Practice guidelines for management of the difficult airway: an updated report by the American Society of Anesthesiologist Task Force on Management of the Difficult Airway. Anesthesiology 2003; 98(5):1269-77.

3. Kesieme EB, Dongo A, Ezemba N, Irekpita E, Jebbin N, Kesieme C. Tube thoracostomy: complications and its management. Pulm Med $2012 ; 2012 ; 256878$.

4. Paolini JB, Donati F, Drolet P. Review article: Video-laryngoscopy: another tool for difficult intubation or a new paradigm in airway management? Can J Anaesth 2013; 60(2):184-91.

5. Aziz MF, Healy D, Kheterpal S, Fu RF, Dillman D, Brambrink AM. Routine clinical practive effectiveness of the Glidescope in difficult airway management: an analysis of 2,004 Glidescope intubations, complications, and failures from two institutions. Anesthesiology 2011; 114(1):34-41. 6. McElwain J, Malik MA, Harte BH, Flynn NM, Laffey JG. Comparison of the C-MAC videolaryngoscope with the Macintosh, Glidescope, and Airtraq laryngoscopes in easy and difficult laryngoscopy scenarios in manikins. Anaesthesia 2010; 65(5):483-9.

7. Mort TC. Tracheal tube exchange: Feasibility of continuous glottis viewing with advanced laryngoscopy assistance. Anesth Analg 2009; 108(4):1228-31.

8. Kadis J, Loskove J. Communicating and Managing the Difficult Airway: One Health Care System's Story. APSF Newsletter 2012; (http:// www.apsf.org/newsletters/html/2012/fall/02airway.htm).

9. Advanced trauma life support for doctors ATLS: manuals for coordinators and faculty. 8th ed. Chicago: American College of Surgeons; 2008. 